\title{
Sexual and reproductive health knowledge, sexual attitudes, and sexual behaviour of university students: Findings of a Beijing- Based Survey in 2010-2011
}

Ming Guan ${ }^{1,2,3}$ (D)

\begin{abstract}
Background: Although several studies have attempted investigating sex-related knowledge, attitudes, and practices among young people in China, deeper insights were still needed to further understand how this population could be supported to make healthy and safe sexual choices. Against this background, this study used a large set of secondary data to examine associations among sexual and reproductive health (SRH) knowledge, sexual attitudes, and sexual behaviour.

Methods: A cross-sectional study was employed to explore the associations among SRH knowledge, sexual attitudes, and sexual behaviour with a publicly available survey data among the 1196 university students from freshmen to Ph.D. candidates. Descriptive analysis was used to describe the sociodemographic characteristics of the university students by gender. Associations of sociodemographic factors with sources and categories of SRH knowledge, categories of contraceptive knowledge, sexual attitudes, and sexual behavior were explored with Poisson regressions and logistic regressions, respectively. The mediating effects of sociodemographic factors on the associations between SRH knowledge and sexual behavior, observed sexual attitudes on the associations of SRH knowledge with sexual behavior, and latent sexual attitudes on the relationship between SRH knowledge and sexual behavior were analyzed in order.

Results: Descriptive analysis showed that the sample was dominated by age group (18 to 24 years), undergraduates, females, limited contraceptive knowledge, unfavorable sexual attitudes, and insufficient knowledge sources. Regression analyses showed that sociodemographic factors had significant associations with SRH knowledge, sexual attitudes, and sexual behavior. Subsequently, the mediating effects of sociodemographic factors on the associations of SRH knowledge and sexual attitudes with sexual behavior were confirmed. Controlling for sociodemographic factors, the effects of sexual attitudes on the associations between SRH knowledge and sexual behaviour could be verified. Structural equation modeling indicated that the linear sequence of sources and categories of SRH knowledge $\rightarrow$ sexual attitudes $\rightarrow$ sexual behaviour model and the triangle mediating effects of
\end{abstract}

Correspondence: gming0604@163.com

'International Issues Center, Xuchang University, Road Bayi 88, Xuchang, Henan, China

${ }^{2}$ Family Issues Center, Xuchang University, Road Bayi 88, Xuchang, Henan,

China

Full list of author information is available at the end of the article

(c) The Author(s). 2021, corrected publication 2022. Open Access This article is licensed under a Creative Commons Attribution 4.0 International License, which permits use, sharing, adaptation, distribution and reproduction in any medium or format, as long as you give appropriate credit to the original author(s) and the source, provide a link to the Creative Commons licence, and indicate if changes were made. The images or other third party material in this article are included in the article's Creative Commons licence, unless indicated otherwise in a credit line to the material. If material is not included in the article's Creative Commons licence and your intended use is not permitted by statutory regulation or exceeds the permitted use, you will need to obtain permission directly from the copyright holder. To view a copy of this licence, visit http://creativecommons.org/ licenses/by/4.0/. The Creative Commons Public Domain Dedication waiver (http://creativecommons.org/publicdomain/zero/1. 0/) applies to the data made available in this article, unless otherwise stated in a credit line to the data. 
sexual behaviour $\rightarrow$ sexual attitudes $\rightarrow$ SRH knowledge model existed.

Conclusions: Sociodemographic factors and observed sexual attitudes mediated the associations between SRH knowledge and sexual behaviour. The sequence relationship: sources and categories of SRH knowledge $\rightarrow$ sexual attitudes $\rightarrow$ sexual behavior and the mediating relationship: sexual behavior $\rightarrow$ sexual attitude $\rightarrow$ sources and categories of SRH knowledge \& sexual behavior $\rightarrow$ sources and categories of SRH knowledge and sexual behavior $\rightarrow$ sexual attitude $\rightarrow$ categories of contraceptive knowledge \& sexual behavior $\rightarrow$ categories of contraceptive knowledge were confirmed in the sample. This study also identified an urgent need for the university students to access to SRH comprehensive knowledge.

Keywords: sexual attitudes, sexual behaviors, sources and categories of SRH knowledge, categories of contraceptive knowledge, mediating relationship, confounding effects, structural equation modeling

\section{Background}

Young people remain a priority population for sexual and reproductive health (SRH) concerns as they continuously account for higher proportions of sexually transmissible infections (STIs) and risky sexual behaviour than other population groups. But, limited knowledge of emergency contraception among university students was reported [1-3]. Moreover, some studies revealed that individual differences in religiosity domains [4], school and parents [5], and sexual behavior of peers [6] influenced adolescents' sexual attitudes and behavioral intent. Till now in China, few studies reflected the possible associations of sociodemographic factors with SRH knowledge, sexual attitudes, and sexual behaviour.

An impressive body of empirical research indicated that insufficient or inaccurate SRH knowledge could lead to negative health outcomes. For example, a study confirmed that inappropriate SRH knowledge might obstruct problematic sexual health behavioral [7]. Without emergency contraception knowledge, risks for unintended pregnancy and reproductive tract infections might be increasing. For example, a study reported that sex without condoms contributed to premarital pregnancy among female undergraduates in China [8]. Additionally, a small but growing body of empirical research revealed unprotected sex in adolescents $[9,10]$ and its adverse effects included depressive symptoms [11] and sexual victimization [12]. To date, the possible relationship between SRH knowledge and sexual behaviour of university students in China was seldom reported.

Several studies indicated that the sexual attitudes could produce change in sexual behavioral among the university students. For example, attitudes towards contraception during adolescence were likely to cause adult contraceptive behavior [13]. Likewise, collegeeducated women were significantly more likely to transition from sexual relationships to cohabiting unions into marriage than less-educated women [14]. In particular, a study suggested that psychological difference between cohabiting and married individuals was confirmed [15].
As to the issues in China, the possible relationship between sexual attitudes and sexual behaviour of university students was needed to further studied.

Some other studies reported the relationship between sexual behavioral and SRH knowledge. For example, knowledge concerning STIs and the risks was insufficient in female Chinese college students [16]. A significant percentage of the college students have poor knowledge, attitude, and practice toward risky sexual behaviors in southwest Ethiopia [17]. Also, the relationship between SRH knowledge and sexual attitudes was documented. For example, a study among school going adolescents reported varied perceptions towards sex education [18]. Regarding these topics in China, the possible associations among SRH knowledge, sexual attitudes, and sexual behaviour were vital to persons accepting higher education.

The aims of this study were to assess the confounding effects of sociodemographic factors on SRH knowledge (sources and categories of SRH knowledge and categories of contraceptive knowledge), sexual attitudes, and sexual behavioral and to determine associations among SRH knowledge, sexual attitudes, and sexual behavioral among the university students with a publicly available survey data. In this study, a series of regressions, mediating analyses, and structural equation models were designed to provide evidences for the associations above. The empirical findings from statistical analyses would provide insights for policy interventions in sexual education for university students.

\section{Literature review}

Knowledge, attitude, and behaviour are vital in the fields of sexual health education. The confounding effects of sociodemographic factors on SRH knowledge, sexual attitudes, and sexual behaviour were documented. For example, age, education, and wealth were significant associated with increase in STIs knowledge [19]. The relevant findings were published globally. 
Inadequate SRH information were reported among refugee adolescent girls in Uganda [20], Kurdish Syrian refugee young women living in Lebanon [21], Mozambican families [22], and adolescent mothers from indigenous populations in Cambodia [23]. Another study among Saudi males indicated that age, education, and income had high impact on contraception awareness and utilization [24]. Consistent with literature, this study therefore hypothesized that:

HO: Sociodemographic factors have significant associations with SRH knowledge, sexual attitudes, and sexual behaviour in China' settings.

The relationships among SRH knowledge, sexual attitudes, and sexual behaviour were documented. For example, a correlation between knowledge, attitude and behavior was confirmed in medical training $[25,26]$. Regarding the relationship between sexual knowledge and behaviour, another study indicated that poor SRH knowledge of male youths increased the risk of female partners' unintended pregnancy in China [27]. Regarding the relationship between sexual attitude and behaviour, a study concluded that psychological and social factors could jointly reduce sexual risk behaviours [28]. Furthermore, several psychosocial predictors of highrisk sexual behaviour were identified [29]. The findings in a nutrition study suggest that gamification dissemination of knowledge could lead to behavioural improvements of adolescents [30]. Clinically, a 'knowledgeattitude-behaviour' sequence was suggested for physician to incorporate clinical practice guidelines into their practice [31]. Similarly, poor level and sources SRH knowledge and perceptions exposed young men to poor sexual outcomes [32]. Accordingly, this study hypothesized that:

H1: The linear sequence of SRH knowledge $\rightarrow$ sexual attitude $\rightarrow$ sexual behavior may exist.

$\mathrm{H} 2$ : The triangle mediating effect of SRH knowledge $\rightarrow$ sexual attitude $\rightarrow$ sexual behavior may exist.

Thus, hypothesized Figures 1 and 2 could be depicted as follow:

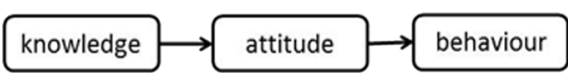

Hypothesized Figure 1. Linear sequence model.

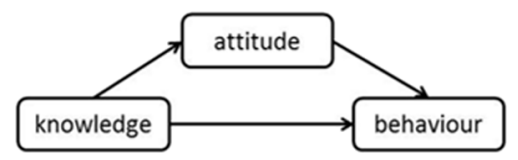

Hypothesized Figure 2. Triangle mediating model.
Combining the logic presented in the rationale for Hypotheses 1 and 2, the Hypotheses with reverse direction could be designed and were depicted as Hypotheses 3 and 4:

H3: The linear sequence of sexual behaviour $\rightarrow$ sexual attitude $\rightarrow$ SRH knowledge may exist.

H4: The triangle mediating effect of sexual behaviour $\rightarrow$ sexual attitude $\rightarrow$ SRH knowledge may exist.

Thus, hypothesized Figures 3 and 4 could be depicted as follow:

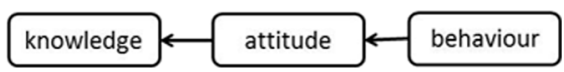

Hypothesized Figure 3. Linear sequence model in reverse direction.

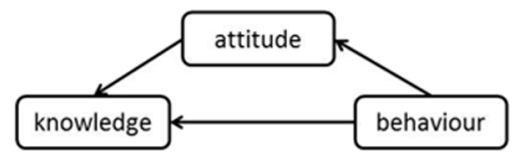

Hypothesized Figure 4. Triangle mediating model in reverse direction.

\section{Methods}

\section{Data source}

Data adopted here were from Survey on the Status Quo of Family Planning Management and Reproductive Health Cognition in College Students (in Chinese: 高校学生计划 生育管理与生殖健康认知现状调查, http://cnsda.ruc.edu. cn/index.php? $r=$ projects/view\&id=76571348) in Beijing in 2010-2011 funded by Institute of health research, School of Social and Population Studies, Renmin University of China. These data have been published by Chinese National Survey Data Archive (CNSDA, http://www. cnsda.org/). CNSDA is an economic and social data sharing platform funded by Key projects of National Natural Science Foundation of China and implemented by $\mathrm{Na}$ tional Survey Research Center at Renmin University of China. Anyone can download the data after registration. The survey questionnaire covered sociodemographic information, sources and categories of SRH knowledge, and health seeking behaviours.

\section{Measures}

\section{Sociodemographic factors}

Here, sociodemographic factors included age group (1518 and $\geq 19$ ), gender (female and male), and educational level (undergraduate level: freshman, sophomore, junior, and senior; postgraduate level: master's candidates and Ph. D candidates). 
Educational duration in China enlightened the author to categorize the age groups. Starting ages in education system in China are generally distributed by 7 years old in primary school (educational time: 5-6 years), 13 years old in junior secondary school (educational time: $3-4$ years), 16 years old in secondary senior school (educational time: 2-3 years), and 19 years old in bachelor's degrees (educational time: 4-5 years). Without compulsory starting age, postgraduate education often initiates after undergraduate education. On the basis of sample characteristics, age groups (15-18, 19-23, and 24-38) were coarsely categorized.

\section{Sources and categories of SRH knowledge}

The variables, accessible knowledge sources, expected knowledge categories, and expected knowledge sources were from three questions, respectively. The first question was: "Which of the following sources do you gain SRH knowledge?" The response options were advertisement leaflets, medical personnel, health educators, school students and friends, TV and magazines, instruction and curriculum, internet, and others. The second question was: "What was expected SRH knowledge of university students?" The response options were sex psychology, venereal disease, contraception, eugenics, sexual behavior, sex ethics, bisexual relationship, birth policy, and genital anatomy. The third question was: "What channels do you want to acquire SRH knowledge?" The response options were advertisement leaflets, lecture series, health educators, medical personnel, school students and friends, TV and magazines, instruction and curriculum, face-to-face consultations, internet, and others. Thus, accessible knowledge sources, expected knowledge categories, and expected knowledge sources could be obtained by summarizing the number of response options, respectively.

\section{Categories of contraceptive knowledge}

Categories of contraceptive knowledge were reflected by three questions: "Do you have the knowledge of emergency contraception?", "Do you have the knowledge of safety period?", and "Do you have the knowledge of condom use?" Their response options were binary values $($ no $=0$, yes $=1$ ).

\section{Sexual Attitudes}

Sexual attitudes included attitudes towards unmarried sex, unmarried pregnancy, and unmarried cohabitation. The three domains were reflected by three questions: "Do you think unmarried sex is acceptable?", "Do you think unmarried cohabitation is acceptable?", and "Do you think unmarried pregnancy is acceptable?", respectively. The response options of first and third questions were "accepted", "depending on the situation", "not accepted", and "do not know". For convenience, the response options were dichotomized into agreement $(=0$, accepted/ depending on the situation/ do not know) and disagreement ( $=1$, not accepted). The response options of second question were "accepted", "not accepted", and "I might as well." Similarly, the response options were grouped into agreement ( $=0$, accepted/ I might as well) and disagreement ( $=1$, not accepted).

\section{Sexual behaviour}

Sexual behaviour was reflected by the question: "Have you ever had sex?" The response options were no $(=0)$ and yes $(=1)$.

\section{Analysis}

There were five stages in the analysis. In the first stage, the descriptive statistics with Chi-square test was used to analyze the distributions of the main variables. In the second stage, associations of sociodemographic factors (age group:, gender, and educational level) with sources and categories of SRH knowledge (accessible knowledge sources: accsour, expected knowledge categories: expcateg, and expected knowledge sources: expsour), categories of contraceptive knowledge (emergency contraception: emerg, safety period: safe, and condom use: condm), sexual attitudes (unmarried sex: sex, unmarried pregnancy: preg, and unmarried cohabitation: cohabit), and sexual behavior (behav) were explored with Poisson regressions and logistic regressions, respectively. In the third stage, generalized linear covariate measurement error models were used to analyze mediating effects of sociodemographic factors on the associations between sources and categories of SRH knowledge and sexual behavior, the associations between categories of contraceptive knowledge and sexual behavior, and the associations between sexual attitudes and sexual behavior with Stata program "cme" [33]. In the fourth stage, the mediating effects of observed sexual attitudes on the associations of sources and categories of SRH knowledge and categories of contraceptive knowledge with sexual behavior were explored with 4-way decomposition using parametric regression models (4wayDPRM) (Stata programme "med4way") [34]. The 4-wayDPRM with delta method standard errors included logistic regression model for the outcome variable (sexual behavior), logistic regression model for the mediators (sexual attitudes: unmarried sex, unmarried pregnancy, unmarried cohabitation), exposure variables (sources and categories of SRH knowledge (knowa) and categories of contraceptive knowledge (knowb)), and covariates (sociodemographic factors: age group, gender, and educational level). In the final stage, structural equation models (SEMs) were conducted to explore mediating effects of latent sexual attitudes on the basis of the hypothesized 
Table 1 Sample characteristics by gender in Beijing, China in 2010-2011

\begin{tabular}{|c|c|c|c|c|}
\hline \multirow[b]{2}{*}{ Age group $(\mathrm{N}=1,100)$} & \multicolumn{2}{|l|}{ Gender } & \multirow{2}{*}{$\begin{array}{l}\text { Chi square } \\
17.4647\end{array}$} & \multirow{2}{*}{$\begin{array}{l}P \text { value } \\
0.000^{* * *}\end{array}$} \\
\hline & Male (\%) & Female (\%) & & \\
\hline $15-18$ & 10.73 & 5.73 & & \\
\hline$\geq 19$ & 40.27 & 43.27 & & \\
\hline Educational level $(N=1,186)$ & & & 9.4684 & $0.002^{* * *}$ \\
\hline Postgraduate & 4.72 & 7.50 & & \\
\hline Undergraduate & 45.87 & 41.91 & & \\
\hline Accessible knowledge sources, median (IQR) & $3(2)$ & $3(2)$ & & \\
\hline Expected knowledge categories, median (IQR) & 3(3) & $4(3)$ & & \\
\hline Expected knowledge sources, median (IQR) & $3(2)$ & $3(2)$ & & \\
\hline Knowledge of emergency contraception $(N=1,176)$ & & & 13.3200 & $0.000^{* * *}$ \\
\hline No & 22.19 & 15.99 & & \\
\hline Yes & 29.17 & 32.65 & & \\
\hline Knowledge of safety period $(N=1,169)$ & & & 6.2467 & $0.012^{* *}$ \\
\hline No & 41.23 & 35.59 & & \\
\hline Yes & 10.44 & 12.75 & & \\
\hline Knowledge of condom use $(N=1,175)$ & & & 134.7675 & $0.000^{* * *}$ \\
\hline No & 36.17 & 17.87 & & \\
\hline Yes & 15.15 & 30.81 & & \\
\hline Unmarried sex $(N=1,187)$ & & & 32.9291 & $0.000^{* * *}$ \\
\hline Agreement & 11.79 & 5.31 & & \\
\hline Disagreement & 38.84 & 44.06 & & \\
\hline Unmarried pregnancy $(N=1,186)$ & & & 22.8226 & $0.000^{* * *}$ \\
\hline Agreement & 22.43 & 15.26 & & \\
\hline Disagreement & 28.16 & 34.15 & & \\
\hline Unmarried cohabitation $(\mathrm{N}=1,184)$ & & & 47.3392 & $0.000^{* * *}$ \\
\hline Agreement & 34.80 & 42.06 & & \\
\hline Disagreement & 15.96 & 7.18 & & \\
\hline Sexual behaviour $(N=1175)$ & & & 72.2138 & $0.000^{* * *}$ \\
\hline No & 47.91 & 37.87 & & \\
\hline Yes & 2.89 & 11.32 & & \\
\hline
\end{tabular}

Note: ${ }^{*}, * *{ }^{* * *}$ denote significance at $10 \%, 5 \%$, and $1 \%$ levels, respectively. IQR interquartile range

relationships among SRH knowledge, sexual attitudes, and sexual behaviour.

The goodness-of-fit for the SEMs with categorical data were determined by indices cutoff criteria: ratio of Chisquare to the number of free parameters $\left(x^{2}: \mathrm{df}\right.$ ratio $<2$ good [35]; < 3 permissible [36]), Tucker-Lewis index (TLI) ( $\geq 0.96)$, comparative fit index (CFI) $(\geq 0.95)$, Standardized Root Mean Squared Error (SRMR) $(<0.08$ [37, 38]), Root Mean Squared Error of Approximation (RMSEA) ( < 0.05: good, 0.05-0.08: adequate, > 0.1: poor) and p of Close Fit (PCLOSE) (> 0.05 [39]), and coefficient of determination (CD). These assessment criteria could be adopted in large sample size ( > 1000) [40]. Here, mediational analysis (including observed and/ or latent variables) was conducted with Stata package medsem [41]. Analysis was conducted in Stata 14.0 for Windows (Stata Corp, College Station, TX, USA).

\section{Results}

The total sample consisted of 1196 respondents. In table 1, basic sample characteristics of university students were reported. The mean age of respondents was 20.75 years (standard deviation $= \pm 1.91$ years) ranging from 15.94 to 37.89 years. Meanwhile, most of the sample was undergraduates $(87.78 \%)$ and females (50.63\%). Simultaneously, the respondents had limited knowledge of emergency contraception (61.82\%), safety period (76.82\%), and condom use (54.04\%). In the meanwhile, part of them accepted unmarried sex (32.77\%), unmarried cohabitation 
Table 2 Incidence rate ratios and odds ratios of sources and categories of sexual and reproductive health knowledge, categories of contraceptive knowledge, sexual attitudes, and sexual behavior in Beijing, China in 2010-2011

\begin{tabular}{|c|c|c|c|c|c|c|c|c|c|c|}
\hline & \multicolumn{3}{|c|}{$\begin{array}{l}\text { sources and categories of SRH } \\
\text { knowledge, IRR( } 95 \% \mathrm{Cl})\end{array}$} & \multicolumn{7}{|c|}{$\begin{array}{l}\text { categories of contraceptive knowledge, sexual attitudes, and sexual behavior ,OR } \\
(95 \% \mathrm{Cl})\end{array}$} \\
\hline & accsour & expcateg & expsour & emerg & safe & condm & sex & preg & cohabit & behav \\
\hline \multicolumn{11}{|l|}{ Age group } \\
\hline $15-18$ & $\begin{array}{l}1 \\
\text { [Reference] }\end{array}$ & $\begin{array}{l}1 \\
\text { [Reference] }\end{array}$ & $\begin{array}{l}1 \\
\text { [Reference] }\end{array}$ & $\begin{array}{l}1 \\
\text { [Reference] }\end{array}$ & $\begin{array}{l}1 \\
\text { [Reference] }\end{array}$ & $\begin{array}{l}1 \\
\text { [Reference] }\end{array}$ & $\begin{array}{l}1 \\
\text { [Reference] }\end{array}$ & $\begin{array}{l}1 \\
\text { [Reference] }\end{array}$ & $\begin{array}{l}1 \\
\text { [Reference] }\end{array}$ & $\begin{array}{l}1 \\
\text { [Reference] }\end{array}$ \\
\hline$\geq 19$ & $\begin{array}{l}1.544^{* * *} \\
\left(1.412^{-}\right. \\
1.687)\end{array}$ & $\begin{array}{l}1.591^{* * *} \\
(1.466- \\
1.726)\end{array}$ & $\begin{array}{l}1.546^{* * *} \\
(1.414- \\
1.690)\end{array}$ & $\begin{array}{l}1.421^{* * *} \\
(1.096- \\
1.844)\end{array}$ & $\begin{array}{l}0.583^{* * *} \\
(0.441- \\
0.771)\end{array}$ & $\begin{array}{l}1.040 \\
(0.792- \\
1.367)\end{array}$ & $\begin{array}{l}2.221^{* * *} \\
(1.641- \\
3.008)\end{array}$ & $\begin{array}{l}1.259^{*} \\
(0.971- \\
1.633)\end{array}$ & $\begin{array}{l}0.452^{* * *} \\
(0.340- \\
0.601)\end{array}$ & $\begin{array}{l}0.526^{* * *} \\
(0.377- \\
0.733)\end{array}$ \\
\hline \multicolumn{11}{|l|}{ Gender } \\
\hline Male & $\begin{array}{l}1 \\
\text { [Reference] }\end{array}$ & $\begin{array}{l}1 \\
\text { [Reference] }\end{array}$ & $\begin{array}{l}1 \\
\text { [Reference] }\end{array}$ & $\begin{array}{l}1 \\
\text { [Reference] }\end{array}$ & $\begin{array}{l}1 \\
\text { [Reference] }\end{array}$ & $\begin{array}{l}1 \\
\text { [Reference] }\end{array}$ & $\begin{array}{l}1 \\
\text { [Reference] }\end{array}$ & $\begin{array}{l}1 \\
\text { [Reference] }\end{array}$ & $\begin{array}{l}1 \\
\text { [Reference] }\end{array}$ & $\begin{array}{l}1 \\
\text { [Reference] }\end{array}$ \\
\hline Female & $\begin{array}{l}1.129^{* * *} \\
(1.053- \\
1.210)\end{array}$ & $\begin{array}{l}1.227^{* * *} \\
(1.153- \\
1.305)\end{array}$ & $\begin{array}{l}1.207^{* * *} \\
(1.125- \\
1.295)\end{array}$ & $\begin{array}{l}1.485^{* * *} \\
(1.162- \\
1.897)\end{array}$ & $\begin{array}{l}1.197 \\
(0.908- \\
1.579)\end{array}$ & $\begin{array}{l}3.692^{* * *} \\
(2.867- \\
4.753)\end{array}$ & $\begin{array}{l}2.627^{* * *} \\
(1.882- \\
3.665)\end{array}$ & $\begin{array}{l}1.698^{* * *} \\
(1.330- \\
2.167)\end{array}$ & $\begin{array}{l}0.390^{* * *} \\
(0.291- \\
0.523)\end{array}$ & $\begin{array}{l}2.603^{* * *} \\
(1.829- \\
3.704)\end{array}$ \\
\hline \multicolumn{11}{|l|}{ Educational level } \\
\hline Postgraduate & $\begin{array}{l}1 \\
\text { [Reference] }\end{array}$ & $\begin{array}{l}1 \\
\text { [Reference] }\end{array}$ & $\begin{array}{l}1 \\
\text { [Reference] }\end{array}$ & $\begin{array}{l}1 \\
\text { [Reference] }\end{array}$ & $\begin{array}{l}1 \\
\text { [Reference] }\end{array}$ & $\begin{array}{l}1 \\
\text { [Reference] }\end{array}$ & $\begin{array}{l}1 \\
\text { [Reference] }\end{array}$ & $\begin{array}{l}1 \\
\text { [Reference] }\end{array}$ & $\begin{array}{l}1 \\
\text { [Reference] }\end{array}$ & $\begin{array}{l}1 \\
\text { [Reference] }\end{array}$ \\
\hline Undergraduate & $\begin{array}{l}1.944^{* * *} \\
(1.787- \\
2.115)\end{array}$ & $\begin{array}{l}2.403^{* * *} \\
(2.223- \\
2.596)\end{array}$ & $\begin{array}{l}1.881^{* * *} \\
(1.728- \\
2.047)\end{array}$ & $\begin{array}{l}1.019 \\
(0.801- \\
1.297)\end{array}$ & $\begin{array}{l}0.410^{* * *} \\
\left(0.318^{-}\right. \\
0.530)\end{array}$ & $\begin{array}{l}0.424^{* * *} \\
(0.327- \\
0.550)\end{array}$ & $\begin{array}{l}1.772^{* * *} \\
(1.348- \\
2.330)\end{array}$ & $\begin{array}{l}1.029 \\
(0.809- \\
1.308)\end{array}$ & $\begin{array}{l}0.894 \\
(0.688- \\
1.161)\end{array}$ & $\begin{array}{l}0.131^{* * *} \\
(0.096- \\
0.179)\end{array}$ \\
\hline Observations & 1,088 & 1,066 & 1,068 & 1,074 & 1,068 & 1,073 & 1,085 & 1,083 & 1,083 & 1,074 \\
\hline
\end{tabular}

Note: ${ }^{*}, * * *$ denote significance at $10 \%, 5 \%$, and $1 \%$ levels, respectively. IRR= incidence rate ratio. OR $=$ Odds $\mathrm{Ratio} . \mathrm{Cl}=\mathrm{confidence}$ interval.. accsour $=$ accessible knowledge sources, expcateg= expected knowledge categories, expsour= expected knowledge sources, emerg= knowledge of emergency contraception, safe= knowledge of safety period, and condm $=$ knowledge of condom use , sex= attitude towards unmarried sex, preg= attitude towards unmarried pregnancy, and cohabit $=$ attitude towards unmarried cohabitation, and behav $=$ sexual behavior

(60.05\%), and unmarried pregnancy (18.72\%). Accordingly, insufficient sexual knowledge and unfavorable sexual attitudes were observed in this sample.

With respect to accessible knowledge sources, the majority of sample $(\mathrm{N}=1191)$ obtained SRH knowledge from internet $(57.60 \%)$, followed by schoolmates and friends (54.24\%), TV and magazines (53.57\%), advertisement leaflets $(40.39 \%)$, instruction and curriculum (35.10\%), health educators (33.33\%), and medical personnel (20.49\%).

Regarding knowledge categories, the majority of sample expected to obtain knowledge of sexual mental health $(64.01 \%, n=1,167)$, followed by relationship between two sexes $(57.24 \%, n=1,167)$, contraception knowledge $(55.70 \%, \mathrm{n}=1,167)$, normal sexual behaviour (54.93\%, $n=1,167)$, prevention from STIs $(51.59 \%, n=$ $1,167)$, eugenics $(42.84 \%, \quad n=1,167)$, sexual ethics (31.79\%, $n=1,167)$, childbearing policy $(21.17 \%, n=$ $1,167)$, and genital anatomy $(15.09 \%, n=1,166)$.

Considering expected knowledge sources, the majority of sample $(N=1168)$ expected to obtain SRH knowledge from series of lectures (44.69\%), followed by health educators $(40.24 \%)$, internet $(44.09 \%)$, instruction and curriculum (35.87\%), TV and magazines (33.65\%), medical personnel (33.30\%), advertisement leaflets (27.31\%), schoolmates and friends (22.17\%), and face-to-face consultation $(18.07 \%)$.
Table 2 reported those students aged $\geq 19$ years were $>1$ times more likely to have sources and categories of SRH knowledge, knowledge of emergency contraception, and favorable attitudes towards unmarried sex and unmarried pregnancy than those students aged $15-18$ years. They were also $<1$ times less likely to have knowledge of safety period, favorable attitude towards unmarried cohabitation, sexual behaviour than those students aged 15-18 years. Females tended to have more sources and categories of SRH knowledge, knowledge of emergency contraception and condom use, attitudes towards unmarried sex and unmarried pregnancy, and sexual behaviour and less favorable attitude towards unmarried cohabitation when compared to males. Meanwhile, undergraduates tended to have more sources and categories of SRH knowledge, less knowledge of safety period and condom use, more favorable attitude towards unmarried sex, and less sexual behaviour as compared to postgraduates.

Table 3 reported mediating effects of sociodemographic factors on the associations of sources and categories of SRH knowledge and categories of contraceptive knowledge with sexual behavior. Mediating effects of sociodemographic factors on the associations between sexual attitudes and sexual behavior could be found in Supplementary Table 1. 
Table 3 Mediating effects of sociodemographic factors on the associations between sources and categories of sexual and reproductive health knowledge, categories of contraceptive knowledge, and sexual behavior in Beijing, China in 2010-2011

\begin{tabular}{|c|c|c|c|c|c|c|}
\hline & Model 1 & Model 2 & Model 3 & Model 4 & Model 5 & Model 6 \\
\hline \multicolumn{7}{|c|}{ Outcome model } \\
\hline accsour & $0.059(0.064)$ & & & & & \\
\hline expcateg & & $-2.078 * *(1.002)$ & & & & \\
\hline expsour & & & $-3.645^{* *}(1.438)$ & & & \\
\hline emerg & & & & $-1.363(4.885)$ & & \\
\hline safe & & & & & $48.388(36.571)$ & \\
\hline condm & & & & & & $3.702(76.517)$ \\
\hline sociod & -5.643 & 265.125 & 276.408 & $241.752(568.280)$ & $2275.436 * * *(0.437)$ & $-12.061(576.327)$ \\
\hline constant & $2.166^{* * *}(0.211)$ & $-196.307^{* * *}(4.715)$ & $-202.345^{* * *}(4.883)$ & $-187.527(439.458)$ & -1860.777 & $4.882(385.066)$ \\
\hline \multicolumn{7}{|c|}{ Indirect effects of covariates via true covariate } \\
\hline accsour & $-0.064^{* *}(0.029)$ & & & & & \\
\hline expcateg & & $2.051^{* *}(0.941)$ & & & & \\
\hline expsour & & & $3.200^{* *}(1.320)$ & & & \\
\hline emerg & & & & 12.794(30.317) & & \\
\hline safe & & & & & 42.823(36.585) & \\
\hline condm & & & & & & $-1.601(76.517)$ \\
\hline \multicolumn{7}{|c|}{ Total effects of covariates } \\
\hline accsour & $-0.006(0.057)$ & & & & & \\
\hline expcateg & & $-0.026(0.467)$ & & & & \\
\hline expsour & & & $0.446(0.631)$ & & & \\
\hline emerg & & & & $11.431(29.388)$ & & \\
\hline safe & & & & & $91.210(.627) 0.000$ & \\
\hline condm & & & & & & $2.100(.227) 0.000$ \\
\hline \multicolumn{7}{|c|}{ True covariate model } \\
\hline \multicolumn{7}{|l|}{ sociod } \\
\hline accsour & $0.011^{* *}(0.005)$ & & & & & \\
\hline expcateg & & $0.008^{* *}(0.004)$ & & & & \\
\hline expsour & & & $0.012^{* *}(0.005)$ & & & \\
\hline emerg & & & & $0.053^{* * *}(0.016)$ & & \\
\hline safe & & & & & $.019(.016)$ & \\
\hline condm & & & & & & $0.133(0.015) 0.000$ \\
\hline constant & $0.699^{* * *}(0.017)$ & $0.700^{* * *}(0.016)$ & $0.696^{* * *}(0.016)$ & $0.697^{* * *}(0.013)$ & .716 & $0.668(0.010) 0.000$ \\
\hline res. var. & $0.000(0.000)$ & $0.003(0.001)$ & $0.003(0.001)$ & $0.002(0.001)$ & $.005(0.000)$ & $0.000(0.000)$ \\
\hline \multicolumn{7}{|c|}{ Measurement model } \\
\hline error var. & $0.196(0.005)$ & $0.194(0.005)$ & $0.194(0.005)$ & $0.195(0.005)$ & $.195(.005)$ & $0.193(0.005)$ \\
\hline reliability & $0.000(0.000)$ & $0.013(0.005)$ & $0.013(0.005)$ & $0.011(0.005)$ & $.026(.001)$ & $0.000(0.000)$ \\
\hline No. of obs & 1171 & 1146 & 1149 & 1156 & 1149 & 1155 \\
\hline log likelihood & -2538.6739 & -2475.2182 & -2479.4629 & -2472.3732 & -2404.8148 & -2413.6262 \\
\hline
\end{tabular}

Note: ${ }^{*}, * * * * *$ denote significance at $10 \%, 5 \%$, and $1 \%$ levels, respectively. sociod $=$ sociodemographic factors, accsour $=$ accessible knowledge sources, expcateg $=$ expected knowledge categories, expsour $=$ expected knowledge sources, emerg $=$ knowledge of emergency contraception, safe $=$ knowledge of safety period, and condm $=$ knowledge of condom use

Controlling for confounding sociodemographic factors, the effects of mediators (sexual attitudes) on the relationship between exposure (sources and categories of
SRH knowledge and categories of contraceptive knowledge) and the outcome variable (sexual behavior) could be calculated with total excess relative risk, excess 
Table 4 The 4-way decomposition using parametric regression models with sexual behaviour and sociodemographic factors in Beijing, China in 2010-2011 (Exposure $\rightarrow$ Mediator)

\begin{tabular}{|c|c|c|c|c|c|c|}
\hline & accsour $\rightarrow$ sex & accsour $\rightarrow$ cohabit & accsour $\rightarrow$ preg & expcateg $\rightarrow$ sex & expcateg $\rightarrow$ cohabit & expcateg $\rightarrow$ preg \\
\hline tereri & $0.032(0.082)$ & $0.051(0.076)$ & $0.002(0.066)$ & $-0.037(0.072)$ & $0.009(0.047)$ & $-0.050(0.049)$ \\
\hline ereri_cde & $0.010(0.086)$ & $-0.121(0.125)$ & $0.056(0.067)$ & -0.074() 0.150 & $-0.077(0.075)$ & $-0.092(0.095)$ \\
\hline ereri_intref & $-0.044(0.076)$ & $0.102(0.065)$ & $-0.062(0.061)$ & $0.016(0.102)$ & $0.056(0.042)$ & $0.042(0.060)$ \\
\hline ereri_intmed & $-0.004(0.007)$ & $-0.009(0.006)$ & $-0.001(0.002)$ & $0.001(0.004)$ & $-0.003(0.002)$ & $0.000(0.001)$ \\
\hline \multirow[t]{2}{*}{ ereri_pie } & $0.070 * *(0.034)$ & $0.079 * *(0.032)$ & $0.008(0.010)$ & $0.021(0.016)$ & $0.032^{* *}(0.016)$ & $0.000(0.001)$ \\
\hline & expsour $\rightarrow$ sex & expsour $\rightarrow$ cohabit & expsour $\rightarrow$ preg & emerg $\rightarrow$ sex & emerg $\rightarrow$ cohabit & emerg $\rightarrow$ preg \\
\hline tereri & $-0.087(0.082)$ & $-0.056(0.066)$ & $-0.061(0.058)$ & $2.924^{* * *}(1.066)$ & $2.656^{* * *}(1.022)$ & $2.916^{* * *}(1.044)$ \\
\hline ereri_cde & $-0.096(0.201)$ & $-0.227^{* *}(0.106)$ & $-0.020(0.083)$ & $0.661(0.822)$ & $3.385^{* * *}(1.236)$ & $2.278^{* *}(1.048)$ \\
\hline ereri_intref & $-0.003(0.149)$ & $0.13^{* *} 6(0.053)$ & $-0.046(0.067)$ & $1.461^{* *}(0.671)$ & $-1.155^{* *}(0.555)$ & $0.495(0.475)$ \\
\hline ereri_intmed & $0.000(0.003)$ & $-0.007(0.005)$ & $-0.001(0.002)$ & $0.588^{* *}(0.295)$ & $0.334 *(0.180)$ & $0.102(0.103)$ \\
\hline \multirow[t]{2}{*}{ ereri_pie } & $0.012(0.015)$ & $0.043^{*}(0.024)$ & $0.006(0.007)$ & $0.214^{*}(0.122)$ & $0.093(0.082)$ & $0.041(0.050)$ \\
\hline & safe $\rightarrow$ sex & safe $\rightarrow$ cohabit & safe $\rightarrow$ preg & condm $\rightarrow$ sex & condm $\rightarrow$ cohabit & condm $\rightarrow$ preg \\
\hline tereri & $1.859^{* * *}(0.619)$ & $1.985^{* * *}(0.648)$ & $2.118^{* * *}(0.659)$ & $4.293^{* * *}(1.396)$ & $4.146 * * *(1.388)$ & $4.443^{* * *}(1.399)$ \\
\hline ereri_cde & $-0.435^{* *}(0.178)$ & $2.643^{* * *}(0.853)$ & $2.327^{* *}(0.968)$ & $0.904(0.965)$ & $5.168 * * *(1.663)$ & $4.002^{* * *}(1.505)$ \\
\hline ereri_intref & $1.918^{* * *}(0.519)$ & $-0.847^{*}(0.448)$ & $-0.219(0.558)$ & $2.807^{* * *}(1.053)$ & $-1.651^{* *}(0.700)$ & $0.297(0.664)$ \\
\hline ereri_intmed & $0.290 *(0.156)$ & $0.133(0.111)$ & $-0.018(0.049)$ & $0.489^{* *}(0.250)$ & $0.560^{* *}(0.267)$ & $0.069(0.155)$ \\
\hline ereri_pie & $0.085^{*}(0.048)$ & $0.056(0.042)$ & $0.028(0.028)$ & $0.092(0.061)$ & $0.069(0.078)$ & $0.075(0.055)$ \\
\hline
\end{tabular}

Note: tereri=total excess relative risk; ereri cde=excess relative risk due to controlled direct effect; ereri intref=excess relative risk due to reference interaction; ereri_intmed=excess relative risk due to mediated interaction; ereri_pie=excess relative risk due to pure indirect effect. 4-wayDPRM=4-way decomposition using parametric regression models. accsour= accessible knowledge sources, expcateg= expected knowledge categories, expsour= expected knowledge sources, emerg= knowledge of emergency contraception, safe= knowledge of safety period, condm= knowledge of condom use , sex= attitude towards unmarried sex, preg $=$ attitude towards unmarried pregnancy, and cohabit= attitude towards unmarried cohabitation

relative risk due to controlled direct effect, excess relative risk due to reference interaction, excess relative risk due to mediated interaction, and excess relative risk due to pure indirect effect.

In table 4, the excess relative risk due to pure indirect effects of accessible knowledge sources $\rightarrow$ attitude towards unmarried sex, accessible knowledge sources $\rightarrow$ attitude towards unmarried cohabitation, expected knowledge categories $\rightarrow$ attitude towards unmarried cohabitation, expected knowledge sources $\rightarrow$ attitude towards unmarried cohabitation, knowledge of emergency contraception $\rightarrow$ attitude towards unmarried sex, and knowledge of safety period $\rightarrow$ attitude towards unmarried sex were significant and less than 1 . The total excess relative risk of knowledge of emergency contraception $\rightarrow$ attitude towards unmarried sex, knowledge of emergency contraception $\rightarrow$ attitude towards unmarried cohabitation, knowledge of emergency contraception $\rightarrow$ attitude towards unmarried pregnancy, knowledge of safety period $\rightarrow$ attitude towards unmarried sex, knowledge of safety period $\rightarrow$ attitude towards unmarried cohabitation, knowledge of safety period $\rightarrow$ attitude towards unmarried pregnancy, knowledge of condom use $\rightarrow$ attitude towards unmarried sex, knowledge of condom use $\rightarrow$ attitude towards unmarried cohabitation, and knowledge of condom use $\rightarrow$ attitude towards unmarried pregnancy were significant and more than 1 . The excess relative risk due to controlled direct effects of expected knowledge sources $\rightarrow$ attitude towards unmarried cohabitation and knowledge of safety period $\rightarrow$ attitude towards unmarried sex were significant and less than 0 , while excess relative risk due to controlled direct effects of knowledge of emergency contraception $\rightarrow$ attitude towards unmarried cohabitation, knowledge of emergency contraception $\rightarrow$ attitude towards unmarried pregnancy, knowledge of safety period $\rightarrow$ attitude towards unmarried cohabitation, knowledge of safety period $\rightarrow$ attitude towards unmarried pregnancy, knowledge of emergency contraception $\rightarrow$ attitude towards unmarried cohabitation, knowledge of emergency contraception $\rightarrow$ attitude towards unmarried pregnancy were significant and more than 1 . The excess relative risk due to reference interactions of knowledge of emergency contraception $\rightarrow$ attitude towards unmarried cohabitation, knowledge of safety period $\rightarrow$ attitude towards unmarried cohabitation, and knowledge of condom use $\rightarrow$ attitude towards unmarried cohabitation were significant and less than 0 , while excess relative risk due to reference interactions of expected knowledge sources $\rightarrow$ attitude towards unmarried cohabitation, 


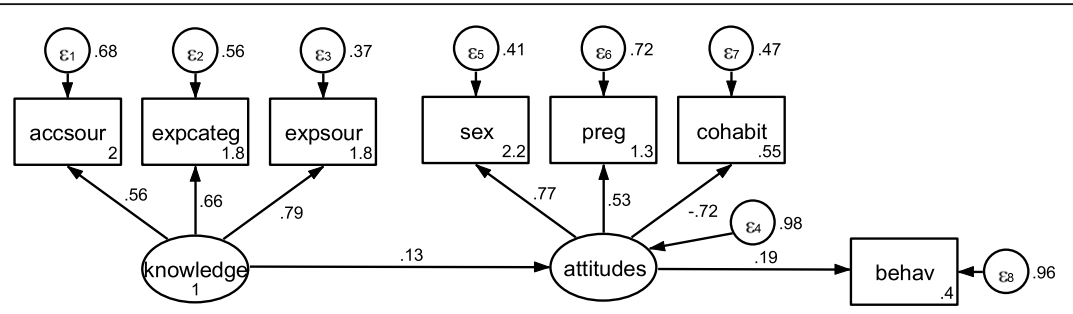

a. Standardized coefficient estimates of hypothesized model 1a.

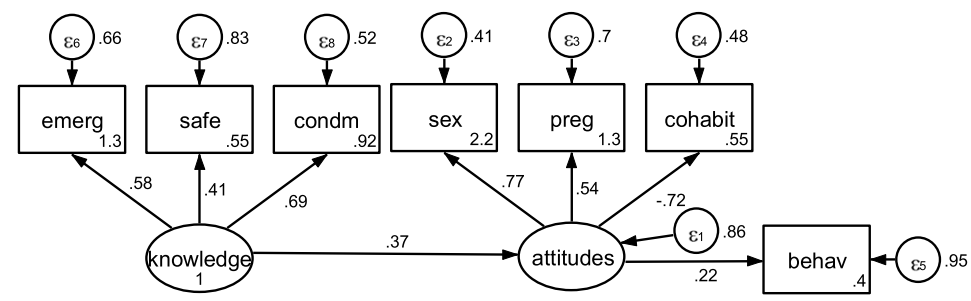

b. Standardized coefficient estimates of hypothesized model $1 \mathrm{~b}$.

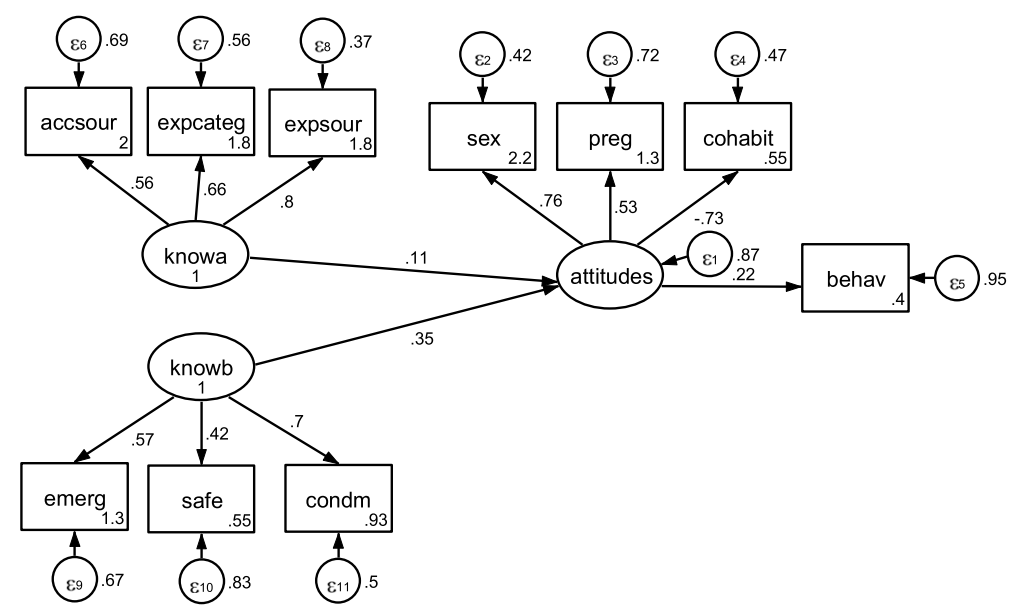

c. Standardized coefficient estimates of hypothesized model 1c.

Fig. 1 a. Standardized coefficient estimates of hypothesized model 1a. b. Standardized coefficient estimates of hypothesized model 1b. c. Standardized coefficient estimates of hypothesized model 1c.

knowledge of emergency contraception $\rightarrow$ attitude towards unmarried sex, knowledge of safety period $\rightarrow$ attitude towards unmarried sex, and knowledge of condom use $\rightarrow$ attitude towards unmarried sex were significant and more than 0 . The excess relative risk due to mediated interactions of knowledge of emergency contraception $\rightarrow$ attitude towards unmarried sex, knowledge of emergency contraception $\rightarrow$ attitude towards unmarried cohabitation, knowledge of safety period $\rightarrow$ attitude towards unmarried sex, knowledge of condom use $\rightarrow$ attitude towards unmarried sex, knowledge of condom use $\rightarrow$ attitude towards unmarried cohabitation were significant, more than 0 , and less than 1 .

Examination of the SRH knowledge $\rightarrow$ sexual attitudes $\rightarrow$ sexual behaviour Model

Standardized coefficient estimates of hypothesized models 1 and 2 could be seen in Figures 1a, 1b, 1c, 2a, and $2 \mathrm{~b}$, respectively. In Table 5 , nearly all of 


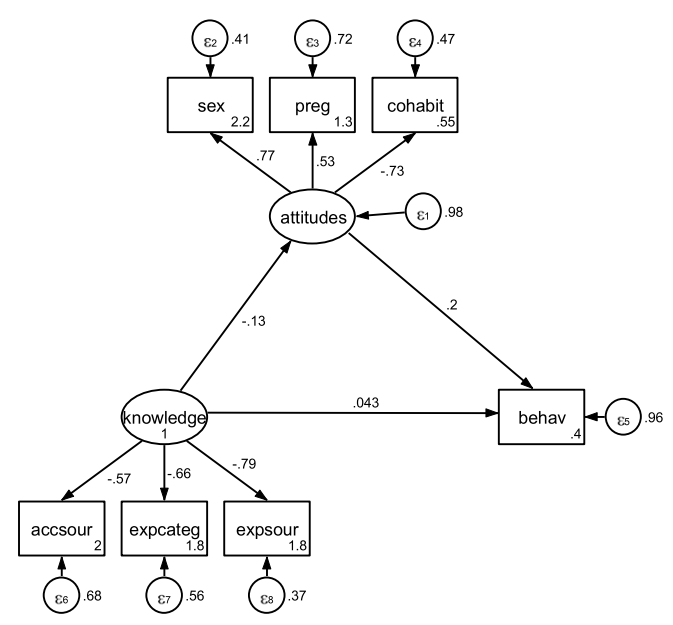

a. Standardized coefficient estimates of hypothesized model $2 \mathrm{a}$

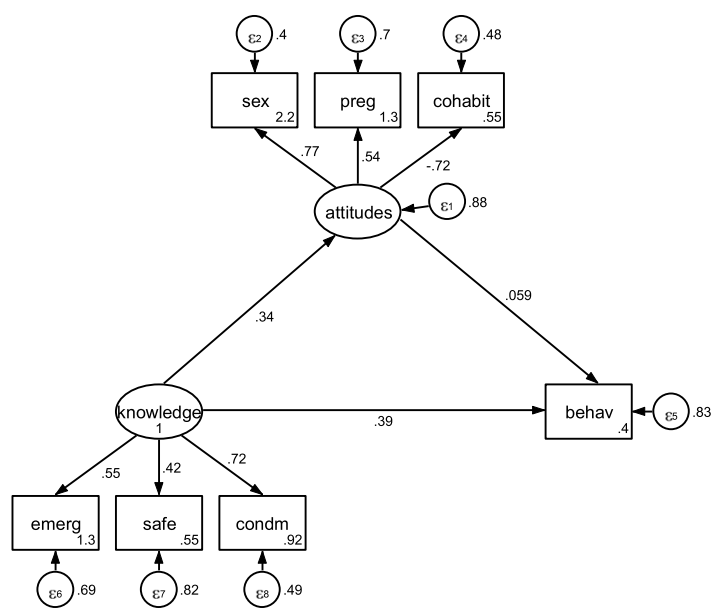

b.Standardized coefficient estimates of hypothesized model $2 b$

Fig. 2 a. Standardized coefficient estimates of hypothesized model 2a. b. Standardized coefficient estimates of hypothesized model 2b

standardized coefficient estimates for SEM in hypothesized models 1 and 2 were significant.

\section{Examination of the sexual behaviour $\rightarrow$ sexual attitudes $\rightarrow$ SRH knowledge model}

Standardized coefficient estimates of hypothesized models 3 and 4 could be seen in Figures 3a, 3b, 3c, 4a, and $4 \mathrm{~b}$, respectively. In Table 6 , all of standardized coefficient estimates for SEM in hypothesized models 3 and 4 were significant.

\section{Assessment on SEM models' fit and indirect effect}

In Table 7, the confirmatory factor analysis of hypothesized models 1a, 2a, 2b, 3c, 4a, and $4 \mathrm{~b}$ gave sufficient goodness-of-fit values: pclose $>0.05, \mathrm{X}^{2}$ : df ratio $<3$, upper bounds of RMSEA $<0.043$, CFI > 0.95, TLI > 0.95 , and SRMR $<0.05$. Due to typographical error and the order of the variables in model $2 a, 2 b$, and $3 c$, the indirect effects could not be calculated. Thus, models 1a, $4 \mathrm{a}$, and $4 \mathrm{~b}$ were considered sufficiently fit.

After calculations, indirect effect (significant estimates, confidence interval (CI), $p<0.001$ ) of Delta's test, Sobel's test, and Monte Carlo's test in model 1a were $0.364(0.318,0.411), 0.364(0.318,0.410)$, and 0.364 $(0.317,0.410)$, respectively. Indirect effect (significant estimates, CI, $p<0.001$ ) of Delta's test, Sobel's test, and Monte Carlo's test in model $1 \mathrm{~b}$ were $0.377(0.330,0.424)$, $0.377(0.332,0.422)$, and $0.377(0.331,0.422)$, respectively. 
Table 5 Standardized coefficient estimates in hypothesized models 1 and 2 in Beijing, China in 2010-2011

\begin{tabular}{|c|c|c|c|c|c|}
\hline & Model 1a & Model 1b & Model 1c & Model 2a & Model 2b \\
\hline \multicolumn{6}{|l|}{ Structural model } \\
\hline attitudes<- knowledge & $0.901^{* * *}(0.008)$ & $0.882^{* * *}(0.012)$ & & $0.901^{* * *}(0.008)$ & $0.872^{* * *}(0.012)$ \\
\hline attitudes<- knowa & & & $0.783^{* * *}(0.021)$ & & \\
\hline attitudes $<-$ knowb & & & $0.434^{* * *}(0.034)$ & & \\
\hline \multicolumn{6}{|l|}{ Measurement model } \\
\hline accsour $<-$ knowledge & $0.936^{* * *}(0.005)$ & & & $0.936 * * *(0.005)$ & \\
\hline expcateg<- knowledge & $0.931^{* * *}(0.005)$ & & & $0.931 * * *(0.005)$ & \\
\hline expsour $<-$ knowledge & $0.945^{* * *}(0.004)$ & & & $0.945^{* * *}(0.004)$ & \\
\hline sex $<-$ attitudes & $0.970^{* * *}(0.006)$ & $0.959^{* * *}(0.007)$ & $0.949 * * *(0.008)$ & $0.970 * * *(0.006)$ & $0.969 * * *(0.007)$ \\
\hline preg<- attitudes & $0.847^{* * *}(0.009)$ & $0.857^{* * *}(0.010)$ & $0.800^{* * *}(0.011)$ & $0.847^{* * *}(0.009)$ & $0.852^{* * *}(0.010)$ \\
\hline cohabit<- attitudes & $0.272^{* * *}(0.029)$ & $0.249^{* * *}(0.029)$ & $0.233^{* * *}(0.025)$ & $0.272 * * *(0.029)$ & $0.247^{* * *}(0.029)$ \\
\hline behav<- attitudes & $0.404^{* * *}(0.026)$ & $0.427^{* * *}(0.025)$ & $0.355^{* * *}(0.023)$ & $0.404 * * *(0.026)$ & $-0.136^{*}(0.076)$ \\
\hline emerg<- knowledge & & $0.896^{* * *}(0.010)$ & & & $0.885^{* * *}(0.010)$ \\
\hline safe $<-$ knowledge & & $0.578^{* * *}(0.022)$ & & & $0.584^{* * *}(0.021)$ \\
\hline condm $<-$ knowledge & & $0.802^{* * *}(0.013)$ & & & $0.811^{* * *}(0.013)$ \\
\hline behav<- knowledge & & & & $0.000(0.000)$ & $0.620^{* * *}(0.076)$ \\
\hline accsour $<-$ knowa & & & $0.931^{* * *}(0.005)$ & & \\
\hline expcateg<- knowa & & & $0.932^{* * *}(0.005)$ & & \\
\hline expsour $<-$ knowa & & & $0.948^{* * *}(0.004)$ & & \\
\hline emerg<- knowb & & & $0.863^{* * *}(0.014)$ & & \\
\hline safe $<-$ knowb & & & $0.602^{* * *}(0.022)$ & & \\
\hline condm $<-$ knowb & & & $0.830^{* * *}(0.015)$ & & \\
\hline Number of obs & 1,128 & 1,137 & 1,119 & 1,128 & 1,137 \\
\hline Log likelihood & -10011.843 & 5457.5305 & -12461.165 & -10011.844 & -5424.9874 \\
\hline
\end{tabular}

Note: ${ }^{* *},{ }^{* * *}$ denote significance at $10 \%, 5 \%$, and $1 \%$ levels, respectively. accsour $=$ accessible knowledge sources, expcateg= expected knowledge categories, expsour= expected knowledge sources, emerg= knowledge of emergency contraception, safe= knowledge of safety period, and condm= knowledge of condom use, sex= attitude towards unmarried sex, preg= attitude towards unmarried pregnancy, and cohabit= attitude towards unmarried cohabitation, and behav= sexual behavior

Indirect effect (significant estimates, CI, $p<0.001$ ) of Delta's test, Sobel's test, and Monte Carlo's test in model 1c were $0.278(0.239,0.317), 0.278(0.240,0.316)$, and $0.278(0.240,0.316)$ with the latent variable: knowa, $0.154(0.125,0.183), 0.154(0.124,0.184)$, and 0.154 $(0.124,0.185)$ with the latent variable: knowb, respectively. Indirect effect of Delta's test, Sobel's test, and Monte Carlo's test in models $3 \mathrm{a}$ and $3 \mathrm{~b}$ were 0.353 (0.311, 0.396), 0.353 (0.312, 0.395), and 0.353 (0.311, 0.394), respectively. Indirect effect (significant estimates, CI, $p<0.001$ ) of Delta's test, Sobel's test, and Monte Carlo's test in model 4 a were $0.306(0.267,0.345), 0.306$ $(0.267,0.346)$, and $0.306(0.266,0.345)$, respectively. Due to indirect effect/total effect $=0.646$, about $65 \%$ of the effect of sexual behaviour on sources and categories of SRH knowledge was mediated by sexual attitudes. Due to indirect effect $/$ direct effect $=1.822$, the mediated effect was about 1.8 times as large as the direct effect of sexual behaviour on sources and categories of SRH knowledge. Indirect effect (significant estimates, CI, $p<$ 0.001) of Delta's test, Sobel's test, and Monte Carlo's test in model $4 \mathrm{~b}$ were $0.353(0.311,0.396), 0.353$ (0.312, $0.395)$, and $0.353(0.311,0.394)$, respectively.

\section{Discussion}

The sample in this study was dominated by age distribution ranging from 18 to 24 years, undergraduate level, limited knowledge of emergency contraception, safety period and condom use, gaps in sources and categories of SRH knowledge, and high prevalence of unfavorable sexual attitudes. This study confirmed that sociodemographic factors had significant associations of with sources and categories of SRH knowledge, categories of contraceptive knowledge, sexual attitudes, and sexual behavior. Subsequently, the mediating effects of sociodemographic factors on the associations of sources and categories of SRH knowledge, categories of contraceptive knowledge, and 


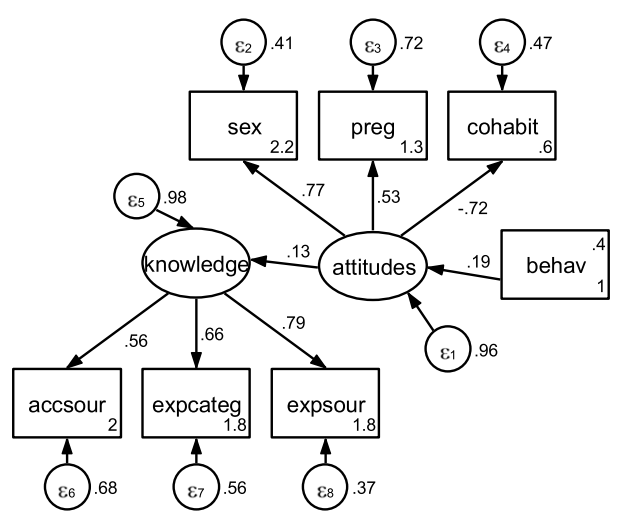

a. Standardized coefficient estimates of hypothesized model 3a

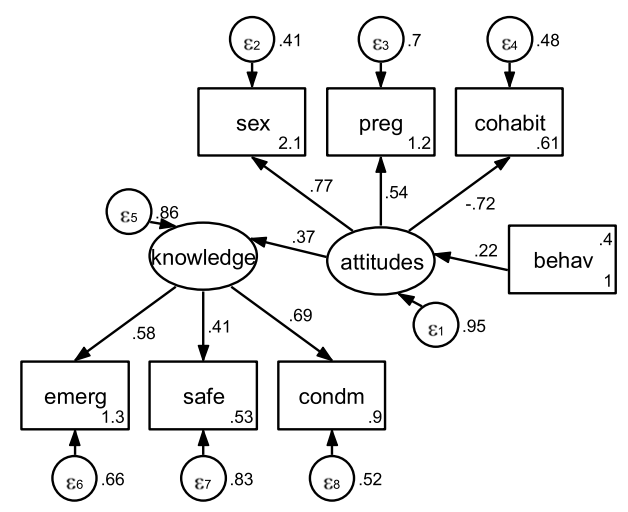

b. Standardized coefficient estimates of hypothesized model $3 \mathrm{~b}$

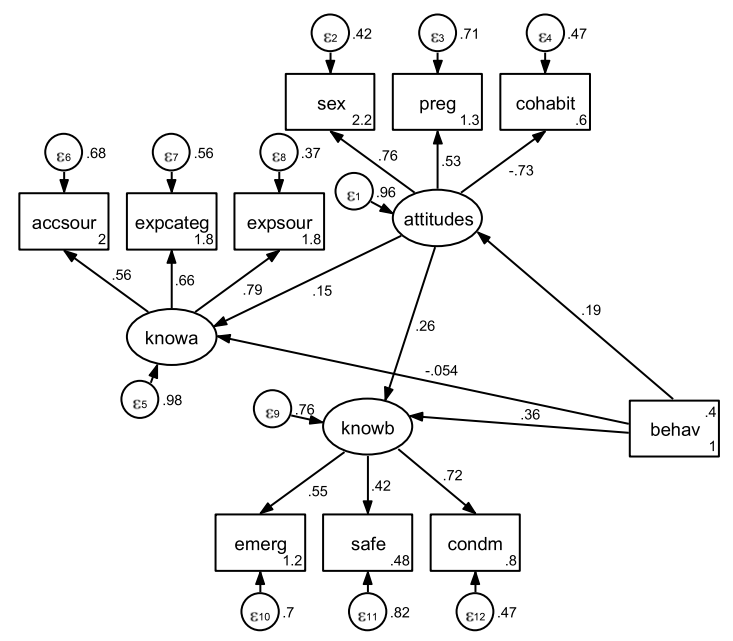

c. Standardized coefficient estimates of hypothesized model $3 \mathrm{c}$

Fig. 3 a. Standardized coefficient estimates of hypothesized model 3a. b. Standardized coefficient estimates of hypothesized model 3b. c. Standardized coefficient estimates of hypothesized model $3 \mathrm{c}$ 


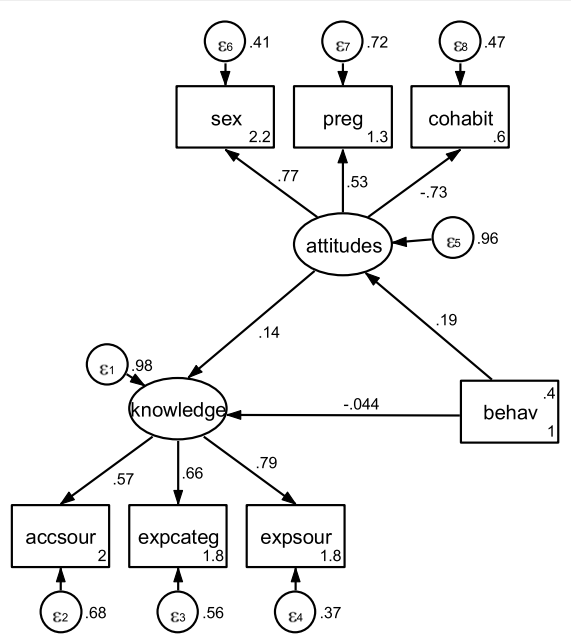

a. Standardized coefficient estimates of hypothesized model 4a

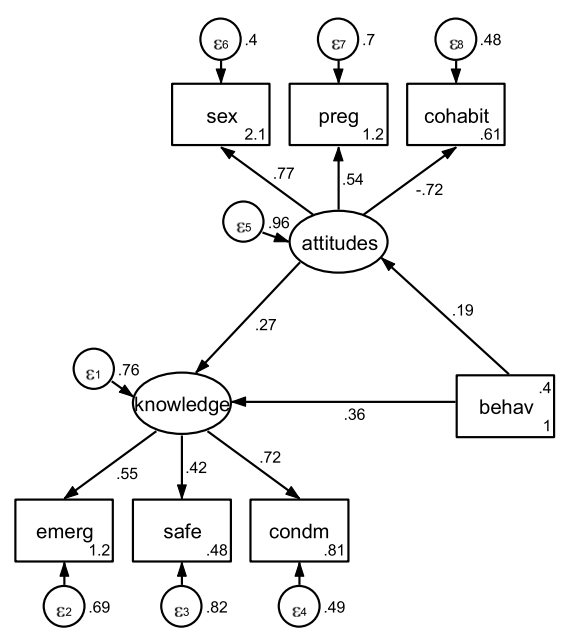

b. Standardized coefficient estimates of hypothesized model $4 \mathrm{~b}$

Fig. 4 a. Standardized coefficient estimates of hypothesized model 4a. b. Standardized coefficient estimates of hypothesized model $4 \mathrm{~b}$.

sexual attitudes with sexual behavior were confirmed. Controlling for sociodemographic factors, the effects of sexual attitudes on the relationship between sources and categories of SRH knowledge and categories of contraceptive knowledge and sexual behavior could be verified. Structural equation modeling indicated that the linear sequence of sources and categories of SRH knowledge $\rightarrow$ sexual attitudes $\rightarrow$ sexual behaviour model and the triangle mediating effects of sexual behaviour $\rightarrow$ sexual attitudes $\rightarrow$ SRH knowledge model existed.

To the best of the knowledge of the author, this was an innovative study to report the associating factors of sources and categories of SRH knowledge among the university students in the capital of China. Beijing, the capital of China, gathers over a hundred of colleges and universities. The findings in this study were in line with several early studies. For example, a cross-sectional survey was conducted at a university in Beijing reported that many students were engaging in premarital sexual intercourse [42]. Likewise, anther cross-sectional survey in Beijing reported that college students with high-risk sexual attitude and behaviors were lack of knowledge and methods [43].

Regarding sociodemographic factors, this study was in congruence with prior studies. Influenced by numerous sociodemographic factors [44], SRH knowledge of college students was mainly from schoolmates and the 
Table 6 Standardized coefficient estimates in hypothesized models 3 to 4 in Beijing, China in 2010-2011

\begin{tabular}{|c|c|c|c|c|c|}
\hline & Model 3a & Model 3b & Model 3c & Model 4a & Model 4b \\
\hline \multicolumn{6}{|l|}{ Structural } \\
\hline knowledge<-attitudes & $0.879^{* * *}(0.012)$ & $0.879^{* * *}(0.012)$ & & $0.910^{* * *}(0.011)$ & $0.805^{* * *}(0.016)$ \\
\hline knowledge<-behav & & & & $-0.024(0.017)$ & $0.168^{* * *}(0.021)$ \\
\hline attitudes<-behav & $0.402^{* * *}(0.024)$ & $0.402^{* * *}(0.024)$ & $0.387^{* * *}(0.024)$ & $0.383^{* * *}(0.024)$ & $0.380^{* * *}(0.024)$ \\
\hline knowa<-attitudes & & & $0.933 * * *(0.010)$ & & \\
\hline knowa<-behav & & & $-0.039 * *(0.016)$ & & \\
\hline knowb<-attitudes & & & $0.830 * * *(0.015)$ & & \\
\hline knowb<-behav & & & $0.154 * * *(0.020)$ & & \\
\hline \multicolumn{6}{|l|}{ Measurement } \\
\hline accsour <-knowledge & & & & $0.935^{* * *}(0.005)$ & \\
\hline expcateg<-knowledge & & & & $0.930 * * *(0.005)$ & \\
\hline expsour $<-$ knowledge & & & & $0.944^{* * *}(0.004)$ & \\
\hline emerg<-knowledge & $0.895^{* * *}(0.010)$ & $0.895^{* * *}(0.010)$ & & & $0.882^{* * *}(0.010)$ \\
\hline safe $<$-knowledge & $0.574^{* * *}(0.022)$ & $0.574^{* * *}(0.022)$ & & & $0.577^{* * *}(0.021)$ \\
\hline condm<-knowledge & $0.799^{* * *}(0.013)$ & $0.799^{* * *}(0.013)$ & & & $0.807^{* * *}(0.013)$ \\
\hline accsour $<-$-knowa & & & $0.937 * * *(0.005)$ & & \\
\hline expcateg<-knowa & & & $0.929 * * *(0.005)$ & & \\
\hline expsour <-knowa & & & $0.943 * * *(0.004)$ & & \\
\hline emerg<-knowb & & & $0.886^{* * *}(0.010)$ & & \\
\hline safe $<-$ knowb & & & $0.573 * * *(0.021)$ & & \\
\hline condm<-knowb & & & $0.806 * * *(0.013)$ & & \\
\hline sex $<$-attitudes & $0.958^{* * *}(0.007)$ & $0.958^{* * *}(0.007)$ & $0.954^{* * *}(0.005)$ & $0.968^{* * *}(0.006)$ & $0.969^{* * *}(0.007)$ \\
\hline preg<-attitudes & $0.854^{* * *}(0.010)$ & $0.854^{* * *}(0.010)$ & $0.844^{* * *}(0.009)$ & $0.845^{* * *}(0.009)$ & $0.849^{* * *}(0.010)$ \\
\hline cohabit<-attitudes & $0.246^{* * *}(0.028)$ & $0.246^{* * *}(0.028)$ & $0.293 * * *(0.028)$ & $0.271^{* * *}(0.028)$ & $0.244^{* * *}(0.028)$ \\
\hline Number of obs & 1,137 & 1,137 & 1,119 & 1,128 & 1,137 \\
\hline Log likelihood & -5373.6336 & -5373.6336 & -11859.676 & -9925.7291 & -5341.0904 \\
\hline
\end{tabular}

Note: ${ }^{*}, * * * *$ denote significance at $10 \%, 5 \%$, and $1 \%$ levels, respectively

Table 7 Assessment on model fit of models 1 to 4 in Beijing, China in 2010-2011

\begin{tabular}{|c|c|c|c|c|c|c|c|c|}
\hline & $x^{2}: d f$ ratio & RMSEA & $90 \% \mathrm{Cl}$ & pclose & $\mathrm{CFI}$ & TLI & SRMR & $C D$ \\
\hline Model 1a & 1.553 & 0.022 & $0.000-0.040$ & 0.997 & 0.995 & 0.992 & 0.020 & 0.747 \\
\hline Model 1b & 8.919 & 0.083 & $0.070-0.098$ & 0.000 & 0.915 & 0.863 & 0.058 & 0.642 \\
\hline Model 1c & 5.207 & 0.061 & $0.052-0.071$ & 0.019 & 0.926 & 0.899 & 0.054 & 0.911 \\
\hline Model 2a & 1.553 & 0.022 & $0.000-0.041$ & 0.995 & 0.995 & 0.992 & 0.018 & 0.747 \\
\hline Model 2b & 1.600 & 0.023 & $0.000-0.041$ & 0.995 & 0.994 & 0.990 & 0.017 & 0.670 \\
\hline Model 3a & 8.919 & 0.083 & $0.070-0.098$ & 0.000 & 0.915 & 0.863 & 0.058 & 0.049 \\
\hline Model 3b & 8.919 & 0.083 & $0.070-0.098$ & 0.000 & 0.915 & 0.863 & 0.058 & 0.049 \\
\hline Model 3c & 2.165 & 0.032 & $0.022-0.043$ & 0.998 & 0.981 & 0.972 & 0.031 & 0.175 \\
\hline Model 4a & 1.553 & 0.022 & $0.000-0.041$ & 0.995 & 0.995 & 0.992 & 0.018 & 0.039 \\
\hline Model 4b & 1.600 & 0.023 & $0.000-0.041$ & 0.995 & 0.994 & 0.990 & 0.017 & 0.173 \\
\hline
\end{tabular}


internet [45]. Also, sexually active adolescents obtained SRH knowledge mainly from peers or mass media rather than teachers and parents [46]. In addition, a prior study indicated SRH knowledge was related to educational attainment [47]. Regarding gender, this study was also consistent with a prior study which highlighted male students' contraceptive responsibilities [48].

Regarding association of contraceptive knowledge with sexual behaviour, this study was in agreement with prior studies. Substantial bodies of current research showed widespread deficits in contraceptive knowledge were in pregnant adolescents [49], tertiary students [50], female undergraduates [51], and teenage mothers [52]. Even worse, medical postgraduates had poor knowledge of condom use [53]. Subsequently, poor contraceptive knowledge resulted in unprotected sexual intercourse [54]. Especially, the students with sexual experience and lack of SRH knowledge would face more risks for sexual health.

Obviously, the relationship of knowledge-attitudebehavior in this study was in line with early studies. For example, a study in public health concluded good knowledge and attitude did not positively translate into good hygiene practices [55]. Another study in medical students indicated that the knowledge could not change the oral health behaviour, but the relationship of behaviour $\rightarrow$ attitude existed [56]. A nutritional study indicated that the relationships of nutrition-related knowledge $\rightarrow$ dietary behavior, nutrition-related attitude $\rightarrow$ dietary behavior, and nutrition-related knowledge $\rightleftarrows$ attitude might exist [57]. But, the relationship of knowledgeattitude-behavior in this study was incongruent with an early study which reported inconformity between attitudes and behaviors and no significant correlations among attitude, behavior, and knowledge among family physicians [58].

Regarding the relationship of sexual behavior $\rightarrow$ SRH knowledge, several facts could help understanding the association. For example, a substantial proportion of out-ofschool youth engaged in risky sexual behaviours without condom use in China [59]. Still, a small proportion of young adults without adequate knowledge of contraception were showing open attitude to premarital sex and engaging in risky sexual behaviours $[60,61]$. Possible reasons for the relationship of sexual behavior $\rightarrow$ SRH knowledge included sexual pleasure [62], condom use experiences [63], and poor self-protection awareness [64].

\section{Policy implications}

Considering policy intervention, this study deepened understanding the role of sexual education on the campus. Simultaneously, this study demonstrated a low level of SRH knowledge and a high proportion of unfavorable sexual attitudes in the sample. Also, some scholars argued that proper SRH education should be implemented to reduce risk sexual behaviors among college students in China $[65,66]$. Thus, health education should help to enhance awareness, enrich information sources and categories, and reduce sexual risk behaviors that caused adverse outcomes.

\section{Conclusion}

In conclusion, the effects of sexual behaviour on sexual attitudes, contraceptive knowledge, and sources and categories of SRH knowledge among the university students were demonstrated. Simultaneously, mediating effects of sexual attitudes on the associations between SRH knowledge and sexual behaviour were confirmed. Accessible sources and categories of SRH knowledge could not meet the demand of the sample. Thus, there was an urgent need for comprehensive sexual education to improve sexual behaviours and modify sexual attitudes through designing sexual education curricula for the university students.

\section{Abbreviations}

SRH: Sexual and reproductive health; STIs: Sexually transmissible infections; IRR: Incidence rate ratio; OR: Odds Ratio; Cl: Confidence interval; CNSDA: Chinese National Survey Data Archive; 4-wayDPRM: 4-way decomposition using parametric regression models; SEM: Structural equation model

\section{Supplementary Information}

The online version contains supplementary material available at https://doi. org/10.1186/s13690-021-00739-5.

\section{Additional file 1}

\section{Acknowledgements}

The author of this paper would like to acknowledge the very helpful comments of the reviewers on the original submission.

\section{Author's contributions}

MG designed the study, performed the statistical analysis, and completed the original version. The author read and approved the final manuscript.

\section{Authors' information}

Ming Guan is heads of the International Issues Center and Family Issues Center at Xuchang University. He is interested in health care service, health change, and quality of life of migrants and elders in the world.

\section{Funding}

This project was funded by Multi-dimensional Evaluation of Health Service System of Floating Population in Henan Province (in Chinese: 河南省流动人 口健康服务体系多维度评价研究; Project number: 2020BSH014) from 2020 Planning of Philosophy and Social Sciences in Henan Province (in Chinese: 2020年河南省哲学社会科学规划年度项目).

This project was also funded by Construction study and practice of ideological and political teaching in the course of China Geography (in Chinese:《中国地理》课程思政建设研究与实践; Project number: 407) from Research and Practice Project of Higher Education \& Pedagogy Reform in Henan Province in 2019 (in Chinese: 2019年度河南省高等教育教学改革研 究与实践项目)

The funding body played no role in the study design, data collection, data analysis, data interpretation and manuscript writing. The content is solely the responsibility of the author and does not necessarily represent the official views of the Henan Provincial Government. 


\section{Availability of data and materials}

http://www.cnsda.org/

\section{Declarations}

\section{Ethics approval and consent to participate}

Not applicable.

\section{Consent for publication}

Not applicable.

\section{Competing interests}

The author declared no potential conflict of interest with respect to the research, authorship and/or publication of this article.

\section{Author details}

${ }^{1}$ International Issues Center, Xuchang University, Road Bayi 88, Xuchang, Henan, China. ${ }^{2}$ Family Issues Center, Xuchang University, Road Bayi 88, Xuchang, Henan, China. ${ }^{3}$ School of Business, Xuchang University, Road Bayi 88, Xuchang, Henan, China.

Received: 24 March 2021 Accepted: 16 November 2021 Published online: 29 November 2021

\section{References}

1. Ahmed FA, Moussa KM, Petterson KO, Asamoah BO. Assessing knowledge, attitude, and practice of emergency contraception: a cross- sectional study among Ethiopian undergraduate female students. BMC Public Health. 2012; 12:110. Published 2012 Feb 9. https://doi.org/10.1186/1471-2458-12-110

2. Tajure N, Pharm B. Knowledge, attitude and practice of emergency contraception among graduating female students of jimma university, southwest ethiopia. Ethiop J Health Sci. 2010;20(2):91-7.

3. Nsubuga $\mathrm{H}$, Sekandi JN, Sempeera $\mathrm{H}$, Makumbi FE. Contraceptive use, knowledge, attitude, perceptions and sexual behavior among female University students in Uganda: a cross-sectional survey. BMC Womens Health. 2016;16:6. Published 2016 Jan 27. https://doi.org/10.1186/s12905-01 6-0286-6

4. Ahrold TK, Farmer M, Trapnell PD, Meston CM. The relationship among sexual attitudes, sexual fantasy, and religiosity [published correction appears in Arch Sex Behav. 2011 Aug:40(4):851]. Arch Sex Behav. 2011:40(3):619-30. https://doi.org/10.1007/s10508-010-9621-4

5. Macdowall W, Jones KG, Tanton $C$, et al. Associations between source of information about sex and sexual health outcomes in Britain: findings from the third National Survey of Sexual Attitudes and Lifestyles (Natsal-3). BMJ Open. 2015;5(3):e007837. Published 2015 Mar 5. https://doi.org/10.1136/ bmjopen-2015-007837

6. Chen AC, Neilands TB, Chan SM, Lightfoot M. Contextual influence of Taiwanese adolescents' sexual attitudes and behavioral intent. Nurs Health Sci. 2016;18(3):355-61. https://doi.org/10.1111/nhs.12278.

7. Zhang XD, Kelly-Hanku A, Chai JJ, Luo J, Temmerman M, Luchters S. Sexual and reproductive health risks amongst female adolescents who use amphetamine-type stimulants and sell sex: a qualitative inquiry in Yunnan. China. Harm Reduct J. 2015:12:34

8. Cao Y, Xiao H, Yan H. Li J1, Li S. Prevalence and sex-related risk factors of premarital pregnancy and reproductive tract infections among female undergraduates in Wuhan, China. Asia Pac J Public Health. 2015;27(2 Suppl): 30S-40S.

9. Yen CF, Cheng CP, Wang SY, Ko NY, Hsu ST. Multidimensional discriminative factors for unprotected sex among adolescents in southern Taiwan. Kaohsiung J Med Sci. 2009;25(4):193-202. https://doi.org/10.1016/S1607-551 X(09)70060-9.

10. Chima Anyanwu F, Ter Goon D, Tugli A, et al. Perception of susceptibility to the negative outcomes associated with unprotected sex among University of Venda students. Pak J Med Sci. 2013;29(6):1306-1310. https://doi.org/10.12 669/pjms.296.4111

11. Zhan W, Shaboltas AV, Skochilov RV, Kozlov AP, Krasnoselskikh TV, Abdala N. Depressive symptoms and unprotected sex in St. Petersburg, Russia. J Psychosom Res. 2012;72(5):371-5. https://doi.org/10.1016/j.jpsychores.2012. 01.015 .
12. Masters NT, George WH, Davis KC, et al. Women's unprotected sex intentions: roles of sexual victimization, intoxication, and partner perception. J Sex Res. 2014;51(5):586-98. https://doi.org/10.1080/00224499.2012.763086.

13. Guzzo KB, Hayford SR. Adolescent Reproductive and Contraceptive Knowledge and Attitudes and Adult Contraceptive Behavior. Matern Child Health J. 2018:22(1):32-40. https://doi.org/10.1007/s10995-017-2351-7.

14. Sassler S, Michelmore K, Qian Z. Transitions From Sexual Relationships Into Cohabitation and Beyond. Demography. 2018;55(2):511-34. https://doi.org/1 0.1007/s13524-018-0649-8

15. van Hedel K, Martikainen P, Moustgaard H, Myrskylä M. Cohabitation and mental health: Is psychotropic medication use more common in cohabitation than marriage?. SSM Popul Health. 2018;4:244-253. Published 2018 Feb 2.https://doi.org/10.1016/j.ssmph.2018.01.001

16. Chen M, Liao Y, Liu J, et al. Comparison of Sexual Knowledge, Attitude, and Behavior between Female Chinese College Students from Urban Areas and Rural Areas: A Hidden Challenge for HIV/AIDS Control in China. Biomed Res Int. 2016:2016:8175921. https://doi.org/10.1155/2016/8175921.

17. Tesfaye Y, Agenagnew L. Knowledge, Attitude, and Practices of Jimma Teacher Training College Students Toward Risky Sexual Behaviors, Jimma. Ethiopia. Sex Med. 2020;8(3):554-64. https://doi.org/10.1016/j.esxm.2020.04 006.

18. Siva VK, Nesan GSCQ, Jain T. Knowledge, attitude and perception of sex education among school going adolescents in urban area of Chennai, Tamil Nadu. J Family Med Prim Care. 2021;10(1):259-64. https://doi.org/10.4103/ jfmpc.jfmpc_1650_20.

19. Finlay JE, Assefa N, Mwanyika-Sando M, et al. Sexual and reproductive health knowledge among adolescents in eight sites across sub-Saharan Africa. Trop Med Int Health. 2020;25(1):44-53. https://doi.org/10.1111/ tmi.13332.

20. Ivanova O, Rai M, Mlahagwa W, et al. A cross-sectional mixed-methods study of sexual and reproductive health knowledge, experiences and access to services among refugee adolescent girls in the Nakivale refugee settlement, Uganda. Reprod Health. 2019;16(1):35. Published 2019 Mar 19. https://doi.org/10.1186/s12978-019-0698-5.

21. Korri R, Froeschl G, Ivanova O. A Cross-Sectional Quantitative Study on Sexual and Reproductive Health Knowledge and Access to Services of Arab and Kurdish Syrian Refugee Young Women Living in an Urban Setting in Lebanon. Int J Environ Res Public Health. 2021;18(18):9586. Published 2021 Sep 11. https://doi.org/10.3390/ijerph18189586.

22. Pires $\mathrm{PH}$, Siemens $\mathrm{R}$, Mupueleque $\mathrm{M}$. Improving sexual and reproductive health knowledge and practice in Mozambican families with media campaign and volunteer family health champions. Fam Med Community Health. 2019:7(4):e000089. Published 2019 Nov 28. https://doi.org/10.1136/ fmch-2018-000089.

23. Kenny B, Hoban E, Pors P, Williams J. A qualitative exploration of the sexual and reproductive health knowledge of adolescent mothers from indigenous populations in Ratanak Kiri Province, Cambodia. Rural Remote Health. 2019: 19(4):5240. https://doi.org/10.22605/RRH5240.

24. Sait M, Aljarbou A, Almannie R, Binsaleh S. Knowledge, attitudes, and perception patterns of contraception methods: Cross-sectional study among Saudi males. Urol Ann. 2021:13(3):243-53. https://doi.org/10.4103/ UA.UA 42 20.

25. Lotfi M, Aghazadeh AM, Asgarpour H, Nobakht A. Iranian nurses' knowledge, attitude and behaviour on skin care, prevention and management of pressure injury: A descriptive cross-sectional study. Nurs Open. 2019;6(4):1600-1605. Published 2019 Sep 12. https://doi.org/10.1002/ nop2.365

26. Odai ED, Azodo CC, Chhabra KG. Disaster Management: Knowledge, Attitude, Behavior, Willingness, and Preparedness among Nigerian Dentists. Prehosp Disaster Med. 2019:34(2):132-6. https://doi.org/10.1017/S1049023X1 9000074.

27. Ding R, Guo C, Song X, Zheng X. Male knowledge, attitude and practice and partner pregnancy among Chinese unmarried youth. PLoS One. 2019; 14(3):e0214452. Published 2019 Mar 26. https://doi.org/10.1371/journal.pone 0214452.

28. Lawal AM, Olley BO. Psychosocial factors predicting risky sexual behaviour among long distance truck drivers in Lagos. Nigeria.SAHARA J. 2017;14(1): 213-21. https://doi.org/10.1080/17290376.2017.1405838.

29. Martin-Smith HA, Okpo EA, Bull ER. Exploring psychosocial predictors of STI testing in University students. BMC Public Health. 2018;18(1):664. Published 2018 May 29. https://doi.org/10.1186/s12889-018-5587-2. 
30. Ezezika O, Oh J, Edeagu N, Boyo W. Gamification of nutrition: A preliminary study on the impact of gamification on nutrition knowledge, attitude, and behaviour of adolescents in Nigeria. Nutr Health. 2018;24(3):137-44. https:// doi.org/10.1177/0260106018782211.

31. Ward MM, Vaughn TE, Uden-Holman T, Doebbeling BN, Clarke WR, Woolson RF. Physician knowledge, attitudes and practices regarding a widely implemented guideline. J Eval Clin Pract. 2002;8(2):155-62. https://doi.org/ 0.1046/j.1365-2753.2002.00337.x.

32. Meena JK, Verma A, Kishore J, Ingle GK. Sexual and Reproductive Health: Knowledge, Attitude, and Perceptions among Young Unmarried Male Residents of Delhi. Int J Reprod Med. 2015;2015:431460. https://doi.org/1 $0.1155 / 2015 / 431460$

33. Rabe-Hesketh S. "CME: Stata program to estimate generalized linear models with covariate measurement error," Statistical Software Components S434701, Boston College Department of Economics, revised 04 Sep 2004; 2003.

34. Discacciati A, Bellavia A, Lee JJ, Mazumdar M, Valeri L. Med4way: a Stata command to investigate mediating and interactive mechanisms using the four-way effect decomposition. International of Epidemiology. 2019 Feb; 48(1):15-20. https://doi.org/10.1093/ije/dyy236.

35. Tabachnick BG, Fidell LS. Using Multivariate Statistics. 5th ed. New York: Allyn and Bacon; 2007

36. Kline RB. Principles and Practice of Structural Equation Modeling (2nd Edition ed.). New York: The Guilford Press; 2005.

37. Schreiber JB, Nora A, Stage FK, Barlow EA, King J. Reporting structural equation modeling and confirmatory factor analysis results: A review. The Journal of educational research. 2006;99(6):323-38.

38. Hu L, Bentler PM. Cutoff criteria for fit indexes in covariance structure analysis: Conventional criteria versus new alternatives. Structural Equation Modeling. 1999:6:1-55.

39. Browne, M.W. and Cudeck, R, "Alternative ways of assessing model fit," In K. A. Bollen \& J. S. Long (Eds.), Testing structural equation models (pp. 136161). Sage, Newbury Park, 1993.

40. lacobucci D. Structural equations modeling: Fit Indices, sample size, and advanced topics. Journal of Consumer Psychology. 2010;20(1):90-8.

41. Mehmetoglu M. medsem: a Stata package for statistical mediation analysis. International Journal of Computational Economics and Econometrics, Inderscience Enterprises Ltd. 2018;8(1):63-78.

42. Zhang L, Gao X, Dong Z, Tan Y, Wu Z. Premarital sexual activities among students in a university in Beijing. China. Sex Transm Dis. 2002;29(4):212-5.

43. Zhou H, Wang XY, Ye F, Gu HH, Zeng XP, Wang Y. Contraceptive knowledge, attitudes and behavior about sexuality among college students in Beijing, China. Chin Med J (Engl). 2012;125(6):1153-7.

44. Zhang D, Bi Y, Maddock JE, Li S. Sexual and reproductive health knowledge among female college students in Wuhan. China. Asia Pac J Public Health. 2010;22(1):118-26.

45. Song $Y$. The sexuality education and attitudes of college students in China. Health Education. 2015;115(1):93-104.

46. Zhang L, Li X, Shah $\mathrm{H}$. Where do Chinese adolescents obtain knowledge of sex? Implications for sex education in China. Health Education. 2007;107(4): 351-63.

47. Feng W, Ren P, Shaokang Z, Anan S. Reproductive health status, knowledge, and access to health care among female migrants in Shanghai. China. J Biosoc Sci. 2005;37(5):603-22

48. Long L, Yuan T, Wang M, Xu C, Yin J, Xiong C, et al. Factors associated with condom use among male college students in Wuhan. China. PLoS One. 2012;7(12):e51782.

49. Villanueva LA, Campos R, del Milagro Pérez-Fajardo M. Contraception knowledge and practices in pregnant adolescents. Ginecol Obstet Mex. 2001 Jun;69:239-42. [Article in Spanish]

50. Roberts C, Moodley J, Esterhuizen T. Emergency contraception: knowledge and practices of tertiary students in Durban. South Africa. J Obstet Gynaecol. 2004;24(4):441-5.

51. Akani C, Enyindah C, Babatunde S. Emergency contraception: knowledge and perception of female undergraduates in the niger delta of Nigeria. Ghana Med J. 2008;42(2):68-70.

52. Ngum Chi Watts MC, Liamputtong P, Carolan M. Contraception knowledge and attitudes: truths and myths among African Australian teenage mothers in Greater Melbourne. Australia. J Clin Nurs. 2014;23(15-16):2131-41.

53. Wang L. Zhang KL.AIDS-related knowledge, condom usage among medical postgraduates. Biomed Environ Sci. 2002;15(2):97-102.
54. Calabretto $\mathrm{H}$. Emergency contraception - knowledge and attitudes in a group of Australian university students. Aust N Z J Public Health. 2009:33(3): 234-9.

55. Pang J, Chua SW, Hsu L. Current knowledge, attitude and behaviour of hand and food hygiene in a developed residential community of Singapore: a cross-sectional survey. BMC Public Health. 2015;15:577. Published 2015 Jun 21. https://doi.org/10.1186/s12889-015-1910-3.

56. Sharda AJ, Shetty S. A comparative study of oral health knowledge, attitude and behaviour of non-medical, para-medical and medical students in Udaipur city, Rajasthan. India. Int J Dent Hyg. 2010;8(2):101-9. https://doi. org/10.1111/j.1601-5037.2009.00393.x.

57. Sharma S, Akhtar F, Singh RK, Mehra S. Relationships between nutritionrelated knowledge, attitude, and self-efficacy among adolescents: A community-based survey. J Family Med Prim Care. 2019;8(6):2012-6. https:// doi.org/10.4103/jfmpc.jfmpc_217_19.

58. Uluç NN, Özdemir Ö. The attitude, knowledge, and behavior of family physicians about childhood asthma in Sakarya province. Turk Pediatri Ars. 2019;54(4):225-237. Published 2019 Dec 25. https://doi.org/10.14744/ TurkPediatriArs.2019.39206.

59. Wang B, Li X, Stanton B, et al. Sexual attitudes, pattern of communication, and sexual behavior among unmarried out-of-school youth in China. BMC Public Health. 2007;7:189. Published 2007 Jul 31. https://doi.org/10.1186/14 71-2458-7-189.

60. Yip PS, Zhang H, Lam TH, et al. Sex knowledge, attitudes, and high-risk sexual behaviors among unmarried youth in Hong Kong. BMC Public Health. 2013;13:691. Published 2013 Jul 29. https://doi.org/10.1186/1471-24 58-13-691.

61. Xu H, Xie J, Xiao Z, et al. Sexual attitudes, sexual behaviors, and use of HIV prevention services among male undergraduate students in Hunan, China: a cross-sectional survey. BMC Public Health. 2019;19(1):250. Published 2019 Feb 28. https://doi.org/10.1186/s12889-019-6570-2.

62. Heeren GA, Jemmott JB 3rd, Mandeya A, Tyler JC. Sexual attitudes and behaviour of university students at a rural South African University: Results of a Pilot Survey. J Hum Behav Soc Environ. 2012;22(8):1021-32. https://doi. org/10.1080/10911359.2012.720909.

63. Pan XH, Cong LM, Ma QQ, Xu GZ, Yu FY, Zou Y. Perception on AIDS infection risk and condom use among 2785 college students having had sexual experience in Zhejiang province. Zhonghua Liu Xing Bing Xue Za Zhi. 2006;27(6):499-502.[Article in Chinese]

64. Lin CL, Ye Y, Lin P, et al. Safe Sexual Behavior Intentions among College Students: The Construction of an Extended Theory of Planned Behavior. Int J Environ Res Public Health. 2021;18(12):6349. Published 2021 Jun 11. https://doi.org/10.3390/ijerph18126349.

65. Sun X, Fan L, Chang C, Shi Y. HIV/AIDS related perception and behavior: A comparative study among college students of different majors in China. Int J Nurs Sci. 2020;7(Suppl 1):S74-S79. Published 2020 Jul 20. https://doi.org/1 0.1016/j.ijnss.2020.07.010

66. Lyu J, Shen X, Hesketh T. Sexual Knowledge, Attitudes and Behaviours among Undergraduate Students in China-Implications for Sex Education. Int J Environ Res Public Health. 2020;17(18):6716. Published 2020 Sep 15. https://doi.org/10.3390/ijerph17186716.

\section{Publisher's Note}

Springer Nature remains neutral with regard to jurisdictional claims in published maps and institutional affiliations.

\section{Ready to submit your research? Choose BMC and benefit from:}

- fast, convenient online submission

- thorough peer review by experienced researchers in your field

- rapid publication on acceptance

- support for research data, including large and complex data types

- gold Open Access which fosters wider collaboration and increased citations

- maximum visibility for your research: over $100 \mathrm{M}$ website views per year

At BMC, research is always in progress.

Learn more biomedcentral.com/submission 